\title{
A Social Network Analysis of Face Tracking in News Videos
}

\author{
Benjamin Renoust \\ National Institute of Informatics \\ and JFLI CNRS UMI 3527 \\ Tokyo, Japan \\ Email: renoust@nii.ac.jp
}

\author{
Thanh Duc Ngo \\ University of Information Technology \\ Vietnam National University \\ Ho Chi Minh City, Vietnam \\ Email: thanhnd@uit.edu.vn
}

\author{
Duy Dinh Le \\ and Shin'Ichi Satoh \\ National Institute of Informatics \\ Tokyo, Japan \\ Email:\{ledduy, satoh\}@nii.ac.jp
}

\begin{abstract}
In the age of data processing, news videos are rich mines of information. After all, the news are essentially created to convey information to the public. But can we go beyond what is directly presented to us and see a wider picture? Many works already focus on what we can discover and understand from the analysis of years of news broadcasting. These analysis bring monitoring and understanding of the activity of public figures, political strategies, explanation and even prediction of critical media events. Such tools can help public figures in managing their public image, as well as support the work of journalists, social scientists and other media experts. News analysis can be also seen from the lens of complex systems, gathering many types of entities, attributes and interactions over time. As many public figures intervene in different news stories, a first interesting task is to observe the social interactions between these actors. Towards this goal, we propose to use video analysis to automatise the process of constructing social networks directly from news video archives. In this paper we are introducing a system deriving multiple social networks from face detections in news video. We present preliminary results obtained from analysis of these networks monitoring of the activity of more than a hundred public figures over a decade of the NHK news archives.
\end{abstract}

Keywords-news analysis; social networks; face detection and tracking; multiplex; dynamic; politics; japan

\section{INTRODUCTION}

In the recent years, the publication of news information has migrated from the traditional means of newspapers, radio, and television to the wider audience offered by the Internet. With the rise of the data-intensive science [1] the analysis and monitoring of news information has given birth to the discipline called topic detection and tracking [2] which aims at segmenting, identifying, and following information, mainly from raw textual information. News analysis is now going beyond and image information is also investigated across all varieties of media [3].

The analysis of news information is key to a wide variety of tasks, from sociology and journalism to politics and economy [4]. It could help the comprehension of users behaviors such as what information a category of users can be exposed to [5]. It could also bring new quantitative tools to overcome the limitations of technocratic measures in the investigation of freedom of information [6]. Even if we know that media competition can lead to a lower quality of information [4], we can hope that public broadcasting services tend to convey an official character, and be a reliable baseline for social analysis.

Social networks analysis directly delivered from video content analysis is the contribution of this paper. The social networks are constructed from face detection and tracking of video content from the NHK News 7 broadcast, and enriched with segmentation and domain knowledge. After discussing the related works in the next section, we will present our data in Section III, with characteristics and preprocessing. Section IV will then present the networks we have extracted, with insights in Section V. Because this paper presents preliminary results, we will discuss our observations and future works in Section VI before concluding.

\section{RELATED WORKS}

Our system focuses on faces detected in news video, and new deep learning approaches are very promising: [7] even reaches better-than-human levels of precision in face recognition. We use a simpler approach inherited from [8] but provide face tracking in return.

Many interesting works approache news analysis in a data intensive way, from text analysis. One of the most impressive approach on exploiting news data comes from [9] in which the authors combine news topic threads and demoscopic information to retrieve videos and generate a new summary video to explain prime ministers' resignations. An NLP framework is designed in [10] to characterize news providers, programs, and newsmakers over many channels. The work from [11] is a notable effort in creating networks from news data. They generate actor-action-object networks over years of news, with great potential for building narrations and understanding of a news landscape.

The relevancy of network modeling for social and political studies does not need to be proven anymore [12], and beyond classical metrics [13], networks have been shown efficient for topics and concept analysis [14] and multiplex networks have been explored to analyse news data [15]. Particularly character networks have been broadly analyzed from literature [16], from TV dramas [17], and even a website is dedicated to the social analysis of Game of Thrones [18].

News data has been one main target for visual analytics applications. Although we do not yet address visualization in this paper, the following examples are all inspiring model to orient our analysis. It is brought to help exploring large transmedia news as in [3] and [19] from which not only text but also visual information is used. Faces are also used in the case of [20], which fuses many criteria and modalities to support user's exploration of stories in the corpus, and introduces a network 


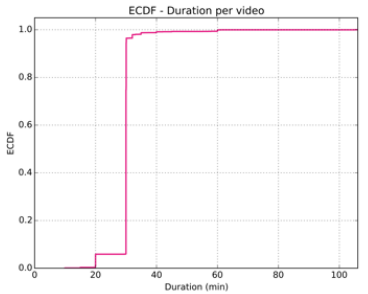

(a) duration per video

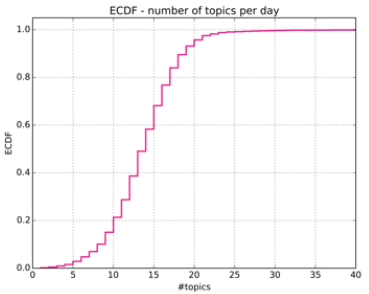

(b) \#topics per day

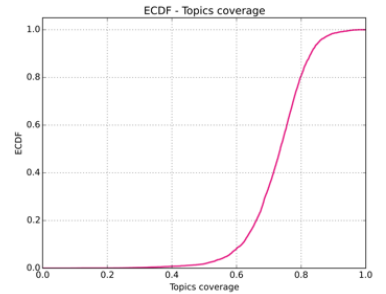

(c) topic coverage

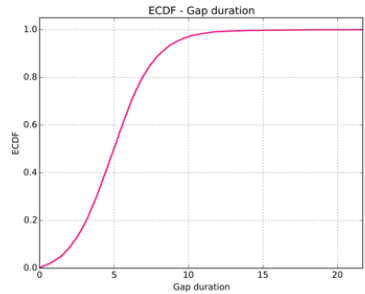

(d) gap duration

Fig. 1. (a) - Most of the programs dure 30 minutes, but some of them may be longer or shorter due to some events like commemorative dates. (b) - We average around 13 topics per day. (c) - Topic segmentation is statistically determined, but most of the news are well covered; only a few topics show large gaps between segmentation (d) so we can expect a good overlap with face tracking.

of topics, similarly to [21]. Analysis derived from large scale data [22] also includes political figures co-occurrence analysis, represented as networks.

\section{FACE DETECTION AND TRACKING}

To understand well the interpretation we can make of the data, we need to draw an accurate picture of what we are looking at. This section details all preprocessing that is done before computing any social network. After describing the data, we introduce the segmentation of news, the face detection and tracking, a some domain knowledge.

\section{A. Description of the data}

Our video dataset consists in the daily-collected NIITVRECS archive from [23]. The capture covers a period between March 17, 2001 and February 27, 2013; of the 4,366day long period, 4,259 news programs have been collected cumulating about 2,102 hours (6.7TB of video) from the NHK channel's daily News 7 broadcast. The few missing captures concerning mostly the beginning of the time period are due to system setup. Most of the programs usually dure $30 \mathrm{mn}$ and only a few of them fall below or beyond this format (news programs may be shorter on Sundays, or longer during commemorative events, see Figure 1(a)).

\section{B. News segmentation}

News are specific programs that can be segmented in different news topics. We thank the authors of [24] for lending us data in which news topics are derived from textual information (captions) synchronized with the news. To summarize the process, a topic boundary corresponds to a point between sentences where the keyword distribution is distinct between preceding and following windows of sentences.

As a result, we have a segmentation of the news by topic, based on semantics analysis (segments in red in Fig. 2-1). Although we do not have the semantic information of these topics (yet), this gives us time boundaries which will turn useful for analyzing people's apparition on screen. Overall, taking into account the differences of lengths among programs, this summarizes in a distribution of an average 13.7 topics per day $(\sigma=4.3)$ as illustrated in Fig. 1(b). However, this segmentation implies that topics are not consecutively segmented, and gaps may occur between two topic detections. So topic detections cover in average $72.6 \%$ of the shows ( $\sigma=0.10$ ), because the beginning (head news summary) and end of the news (weather reports) are ignored by the topic segmentation (Fig. 1(c)). In addition gaps between news topic average 5.1 seconds $(\sigma=2.4)$ and can stretch up to 20,7 seconds (see Fig. 1(d)), in which faces may still be detected. Additionally, a standard shot segmentation derived from color histograms thresholds is provided to help the face-tracking process (segments in blue in Fig. 2-1). This is a contiguous segmentation, without gap in-between corresponding to video cut editing.

\section{Face-track extraction}

Now we can extract faces from the video shots as in [25], roughly decomposed in the following steps (illustrated in Figure 2, steps 2 to 5):

Detection. We first apply a detection of faces in all images, using off-the-shelves techniques such as the Viola-Jones face detector [8] (available from openCV [26]). This incidentally results in a feature space describing each detected face. To reduce the number of false positive results (detected faces that are not actually faces) we set the minimum size of a face at $60 \times 60$ pixels.

Tracking. We now need to group together the detected faces of a same individual into one single face-track. This is done with by generating tracking points within detected faces - a tracking point is a same point identified across different frames, we generated them using the KLT point tracker [27]). Tracking points can belong to one detected face, or to (at least) two faces, or to the background. Based on a confidence grouping measure, these points are differentiated and matched from a starting image with the following image given their temporal order. The process has also been made robust against distortions such as flash lights and occlusions [25]. This results in multiple face instances regrouped in face-tracks. There can be multiple face-tracks across a same video.

Sampling the face-tracks. For each face-track, we create a mean face that is a representative face in the image feature space, based on the k-Faces method [25]. To do so, the facetrack is divided in $k$ sub-tracks of equal size. For each the (temporal) middle face is taken, altogether forming a set of $k$-Faces. The mean face is then a mean point in the feature space described by the $k$-Faces. Hence, $k$ influences sampling, with a larger $k$ for better representativeness (i.e. the average distance between a mean face representing a face-track and all faces in the track). To ensure a best quality of the output, we use $k=20$.

Matching the face-tracks. Face-tracks can finally be matched based on their mean face euclidian distance in the 


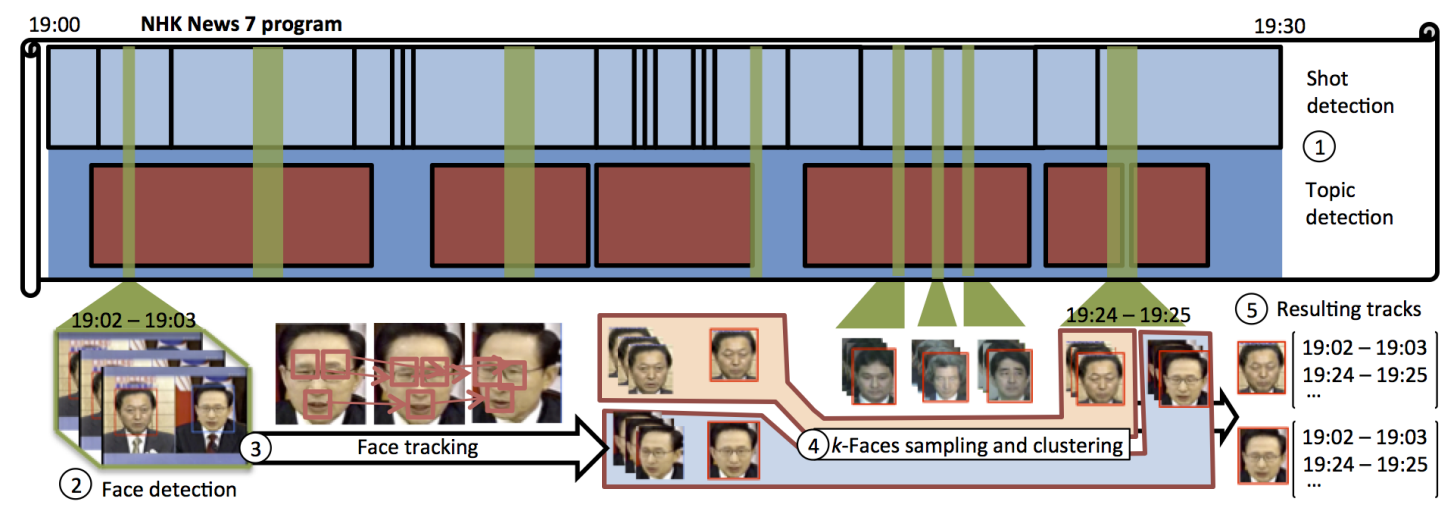

Fig. 2. The overall framework of a news video analysis. (1) The video is segmented into topics (red) and shots (blue). (2) Faces are detected in each frame. (3) Track points are inserted and matched across faces creating tracks. (4) Tracks are sampled and clustered to obtain the final face-tracks for each individual (5).

\section{feature space.}

The whole process has detected over 30 million faces and 174,778 face-tracks were extracted. We need now to identify and recognize groups of face-tracks, and clustering appears as the natural following step. However, clustering implies many new issues that we have not yet addressed this work (but we include this goal as par our our future works section VI). Yet, we can still use a different approach to construct our networks, that is of face retrieval.

The faces of 139 characters have been annotated during the evaluation campaign of [25] giving a ground truth for retrieving matching face-tracks. These faces are the faces of well known people among the Japanese media scene, including celebrities and politicians (Japanese and international), for which we had the highest precision of retrieval and identification. In total, over 5 thousands face-tracks were annotated, and 16,714 facetracks of the 139 different characters were retrieved. This corresponds to 2,984 days of news program over the whole archive having matching persons, covering a total of 36 hours of face tracks.

The coverage of the face-tracks averages $2.4 \%$ of a program ( $\sigma=2.6$ ), reaching the maximum of $38.9 \%$ of a program. The ground truth has been provided during the 2010 period, for which the face-tracks appear slightly denser (3.3\% on average). The average screen apparition per person is $15.7 \mathrm{~min}(\sigma=$ 32.5 ) but there is a lot of variation between people (actually it fits a lognormal distribution Fig. 3(a)), and a few people seem to hold most of the screen time (Table I).

As a result, the tracks work as follows: everyday, we have a news broadcast, and every broadcast contains news segments (topics) and face-tracks of different people. We then observe an average of 23.8 seconds of cumulated detections per topics $(\sigma=28.9$, with a maximum of 383.0 - Fig. 3(b)), with in average 1.28 persons detected per topic $(\sigma=0.66$, with a maximum of 7 - Fig. 3(c)). Thankfully, this shows that we can reasonably expect people to overlap across topics, although $80 \%$ of our topics do not show more than one person detected (see Figure 3(c)). Looking closer at the distribution of interday occurrences of people in topics, we can see that most of them appear on screen mostly on a daily basis, with bigger gaps then (Fig. 3(d) is an example). This is sometimes referred as a characteristic of "bursty" data [28], meaning that over the whole period of time, the is a lower probability for two persons to be detected together than by random, making these links very interesting. We can also notice that most of the people we are tracking seem to take part in similar topics during the 2008-2011 period.

\section{About the 139 persons}

Some background information is necessary for a good understanding of this news data. With a little domain knowledge, we have classified the 139 characters identified into 9 categories depending on what brought them under the light of news: Politics (71), Sports (27), Culture (11), Business (7), Imperial family (5), Journalism (4), Religion (3), Law (3), and Other (5). Additionally, we have enriched them with their country: 96 individuals are from Japan, among which 39 political figures and 22 athletes.

Incidentally, the Politics class includes 23 international leaders (presidents, prime ministers...). The Japanese prime ministers - hereafter referred as PM - governing during the whole period of capture are of course represented, allowing us to create time frames covering their cabinet(s). Yoshiro Mori was the first PM in the timeline, but his mandate only covers a few weeks from the beginning of the capture, so himself is not included in the persons subset. Finally, we obtain 11 time periods (Fig. 9 details them in chronological order, note that the numbers following a PM's name represent different cabinets formed by the same PM). The different time bar charts we present in this paper reflect these different periods as colored backgrounds, as for example in the timelines of the different PM in Figure 5

Although Pearson's correlations between the three measures (Screentime S, \#days D, and \#topics $T$ - per person) are very high $(S-D=0.95, S-T=0.96$, and $D-T=0.97)$, we can use the ranking of the top 10 percentile to extract persons of interest (as presented in Table I).

A background checking gives us supplemental information explaining their occupation of screen space. Out of the obvious known figures and the aforementioned PM, I. Ozawa,S. Maehara, K. Shii, and K. Okada are famous politicians. S. Takeda and $S$. Nakarai are two presenters from NHK. T. Horie is a businessman, H. Matsui is a baseball player, and W. Abe is active on the music scene. 


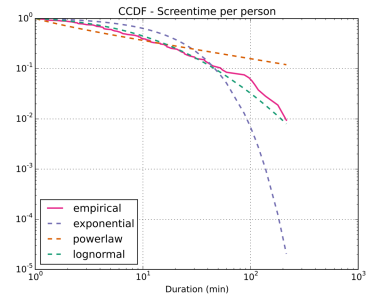

(a) Screen time per person

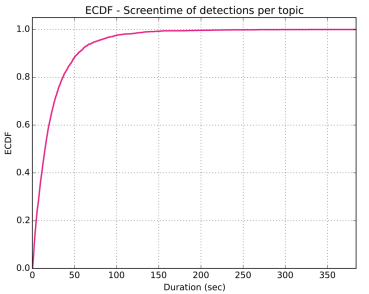

(b) Screen time per topic

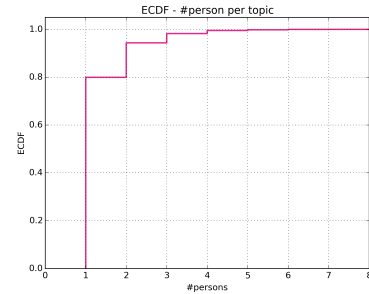

(c) \#persons per topic

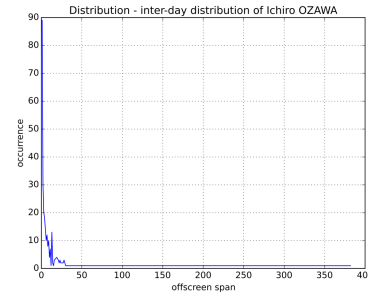

(d) Inter-day distribution of I. Ozawa

Fig. 3. (a) - The distribution of screen time per person fits a log-normal distribution and shows a few people actually holds most of the total screen time. (b) - Face detections in a topic usually average a total of about $24 \mathrm{~s}$. (c) - $80 \%$ of the topics detect only one person. (d) - The distribution of time (in days) between two appearances of I. Ozawa, which is of one day most of the time, is characteristic of the "bursty" behavior of the data.

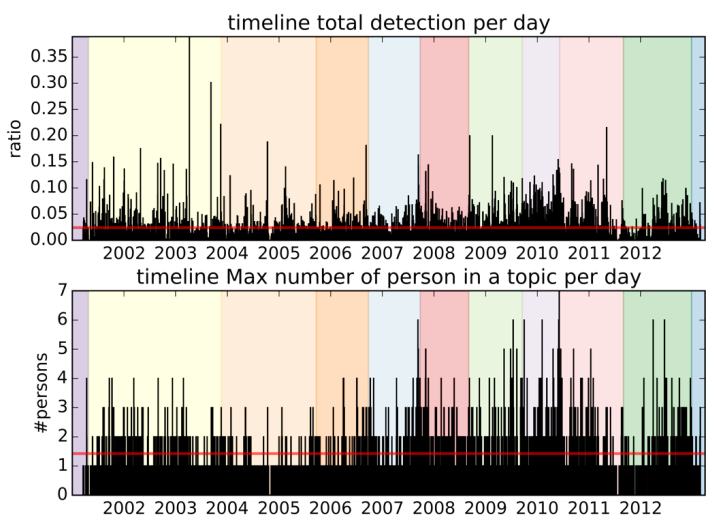

Fig. 4. (Top) The daily track averages $2.4 \%$ of a program (line in red) and shows a bit more coverage during the 2008-2011 period. (Bottom) Even if a lot of topics detect one person only, the maximum detections in a topic per day shows many topics going beyond, especially during the 2008-2011 period.

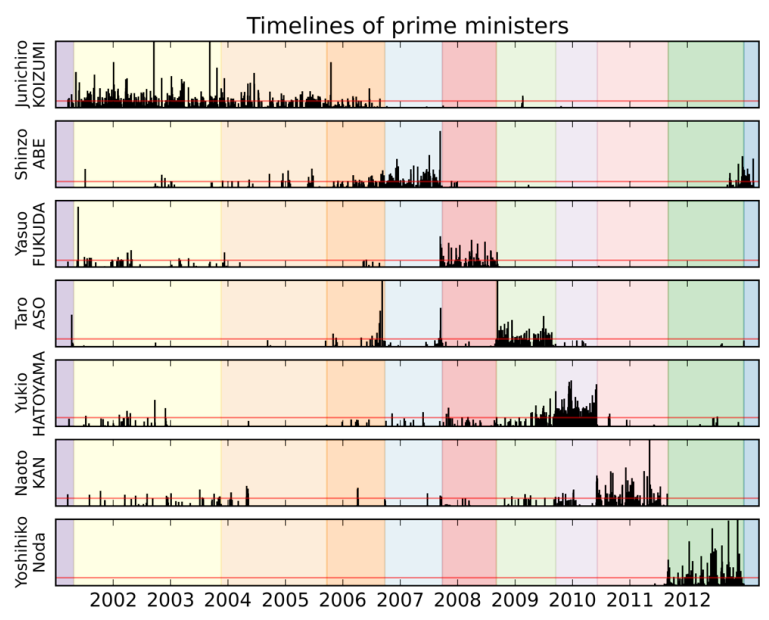

Fig. 5. The different time lines for each of the PM reflects well their mandate (as presented in the background colors). Notice the differences in patterns of time apparition for each PM, particularly Y. Noda who only appeared during his cabinet.

\begin{tabular}{c|c|c|c} 
Person & Screentime & \#Days & \#Topics \\
\hline Junichiro KOIZUMI & $\mathbf{2 1 5}$ & $\mathbf{5 1 6}$ & $\mathbf{5 2 3}$ \\
Yukio HATOYAMA & $\mathbf{1 7 9}$ & $\mathbf{3 3 0}$ & $\mathbf{3 6 8}$ \\
Ichiro OZAWA & $\mathbf{1 3 8}$ & $\mathbf{2 9 4}$ & $\mathbf{3 0 4}$ \\
Naoto KAN & $\mathbf{1 1 8}$ & 243 & 249 \\
Shinichi TAKEDA & $\mathbf{1 1 0}$ & $\mathbf{3 3 7}$ & $\mathbf{4 9 1}$ \\
Shinzo ABE & 103 & 281 & $\mathbf{2 9 8}$ \\
Yoshihiko NODA & 97 & 195 & 245 \\
Taro ASO & 87 & 181 & 187 \\
Yasuo FUKUDA & 60 & 144 & 139 \\
Seiji MAEHARA & 55 & 116 & 126 \\
Takafumi HORIE & 52 & 116 & 121 \\
George BUSH & 45 & 86 & 93 \\
Kazuo SHII & 42 & 116 & 98 \\
Sae NAKARAI & 40 & $\mathbf{3 1 7}$ & 190 \\
Katsuya OKADA & 37 & 115 & 109 \\
Hideki MATSUI & 26 & 126 & 73 \\
Wataru ABE & 22 & 84 & 104 \\
THE TOP 10\% PEOPLE AMONG THE DIFFERENT CRITERIA
\end{tabular}

TABLE I. THE TOP $10 \%$ PEOPLE AMONG THE DIFFERENT CRITERIA (IN BOLD, THE PMS, AND TOP 5 SCORES OF EACH CRITERION). ICHIRO BEEN A PM, NOR IS A NEWS PRESENTER.

\section{DIFFERENT NETWORKS}

In this section we will define and present our different networks with their preliminary analysis. From this point on, we will mostly focus our interpretations on the political scene, and use the networks as its mean of understanding. Most of the following networks use the persons as the same set of nodes, but with different families of ties.

\section{A. Network of people overlapping on screen}

Our first network connects two persons when two facetracks overlap in time. This means that we create a link between two persons when they have been detected simultaneously on screen. These links are enriched with the screen duration of the overlapping of tracks as weights.

This network presents 35 nodes and 44 edges, with a main connected component of 29/41 (Fig. 6, left). This connected component is only composed of politicians, with one business person (M. Shirakawa, connected to Y. Hatoyama). It's worth noting that $J$. Koizumi, the top individual among all other metrics, only presents here a degree of 2 . Four nodes stand out in terms of betweenness centrality (S. Abe:0.16, I. Ozawa:0.14, Y. Hatoyama:0.18, and Y. Noda:0.15, with the rest of the dataset below 0.09), and 2 nodes in terms of degree $(Y$. Hatoyama:10 and Y. Noda:7), however no clear convincing cut of communities is shown by Louvain's algorithm [29].

A few links stand out in terms of screen duration (over 1000), connecting: Y. Noda and S. Tanigaki, in 2012, I. Ozawa 

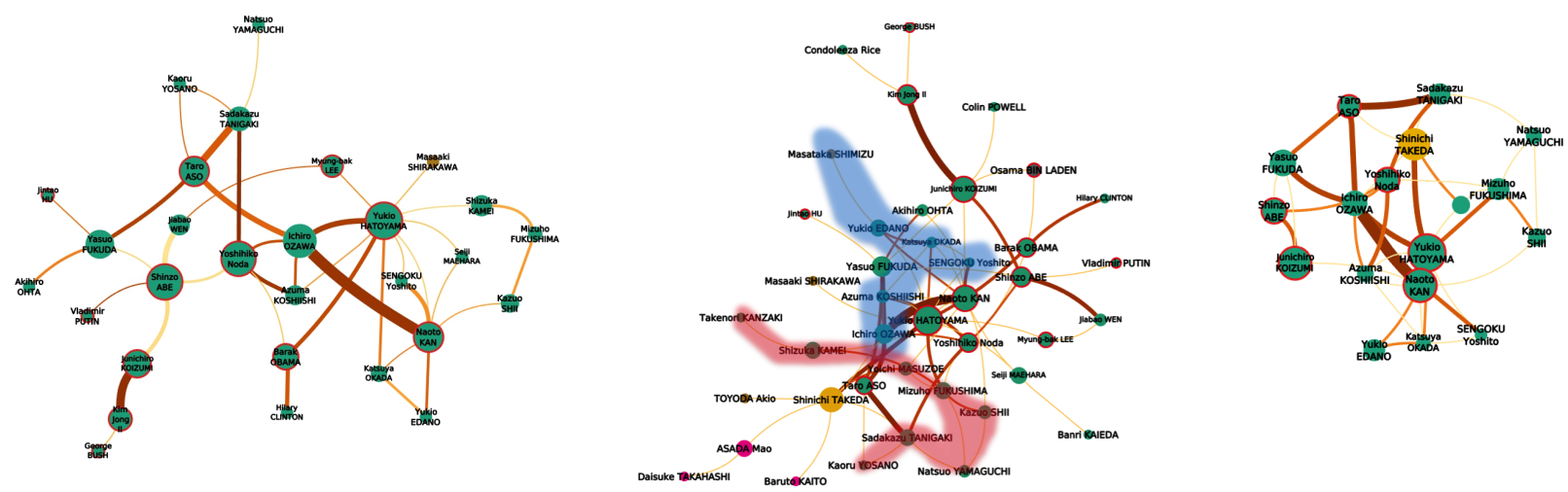

Fig. 6. The pictures better seen zoomed. Green: politicians,brown: businessmen, yellow: journalists, pink: athletes, purple: imperial family. Circled in red are world leaders and PM. The size of the node reflects its betweenness centrality. From light yellow to dark orange, the edges color and width encode their weight. (Left) The network of persons overlapping on screen. (Center) The network of persons appearing on a same shot, with two communities in the colored areas. (Right) The maximum $k$-core community $(k=3)$.

and N. Kan in 2003, 2006, and 2010, Y. Hatoyama and I. Ozawa in 2006, 2010, and 2012, Y. Hatoyama and B. Obama in 2009, J. Koizumi and Kim Jong Il 2002, V. Puttin and S. Abe in 2012, T. Aso and Y. Fukuda in 2009. When looking at the number of days in which two different persons appear together, we can notice stronger links between: $S$. Tanigaki and T. Aso in 2006, J. Koizumi and S. Abe in 2002, and H. Clinton and B. Obama in 2008.

\section{B. Network of people appearing in a same shot}

This second family of ties defines links between people appearing in a same shot (i.e. an uncut segment of video). This network roughly extends the previous network, with the difference that people do not need to appear on screen together. Because shot duration greatly varies depending on the cut of the video, we cannot use it as a meaningful metric to weigh edges, instead, we will consider the number of different days that include these shots.

The network (Fig. 6, center) presents 49 nodes for 75 edges with a main component of $41 / 71$. The maximum $k$-core $(k=3)$ [30] presents a very intricate subnetwork of 18 nodes (Fig. 6, right). It includes the PM, and the main anchorman ( $S$. Takeda), later referred as the 'main actors'. All the other nodes are politicians, including I. Ozawa. Getting their full list and description may go beyond the scope of this paper, but it is interesting to notice that $N$. Yamaguchi stands out as the only politician not directly connected to any of the PM. The main component presents a wider range of types of people, including 3 athletes, 3 business persons, and $O$. Bin Laden. A Louvain segmentation does not present a clear cut of denser subgroups in this network. If we remove the 'main actors', we can interestingly observe two communities of politicians (the colored areas in Fig. 6, center), one centered on M. Fukushima and N. Yamaguchi, and the other one on K. Okada. However one should carefully interpret the meaning of these links given the low amount of common shots (at most three).

Three edges stand out with links displaying between 5 and 8 days of connections, T. Aso and S. Tanigaki, N. Kan and Y. Hatoyama, J. Koizumi and Kim Jong Il. If we consider links connecting two persons over one day only as 'casual' and discard them, we can reveal a network of stronger ties of persons with 'recurrent' interactions (23/26). In this network,
I. Ozawa displays the highest betweenness centrality, followed then by the different PM.

\section{Networks of people appearing during a same topic}

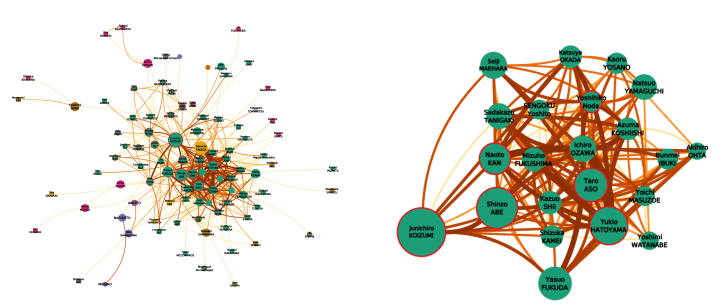

Fig. 7. Pictures better seen zoomed. Same encoding as in Fig. 6. Edge weight corresponds to the number of common topics. (Left) The network of persons detected during a same topic. (Right) The $k$-core $(k=13)$ of this network.

The following network connects individuals when they have been detected during a same topic, based on the segmentation described in Section III-B. This means that two persons are connected when they took part of a same media event. The graph connects 107 people over 507 links with a main connected component of 96/499 (Fig. 7, left). This graph presents characteristics closer to complex networks with a long tail distribution of node degrees (actually fitting a lognormal distribution).

Knowing that co-detection during a news topic is the reason linking nodes, we should first remove the journalists - occurring a lot in the dataset, in order to focus on other people's interactions. The resulting graph presents a maximal $k$-core $(k=12)$ gathering 15 Japanese politicians and the 7 $\mathrm{PM}$ in a subgraph $G_{k=12}^{\prime}$ with a density $D_{G_{k=12}^{\prime}}=0.79$ (Fig. 7, right).

A degree and a centrality analysis will bring focus to the same people identified in the previous networks. To go beyond, we will look at the graph without the 'main actors', leaving 67 nodes for 221 edges. This graph clearly presents community structures, and by running a Louvain algorithm, we obtain a very interesting clustering result. The two main partitions (in light green and orange in Fig. 8) clearly present international politicians and national politicians (respectively). We are now able to spot the non-PM Japanese politicians who 


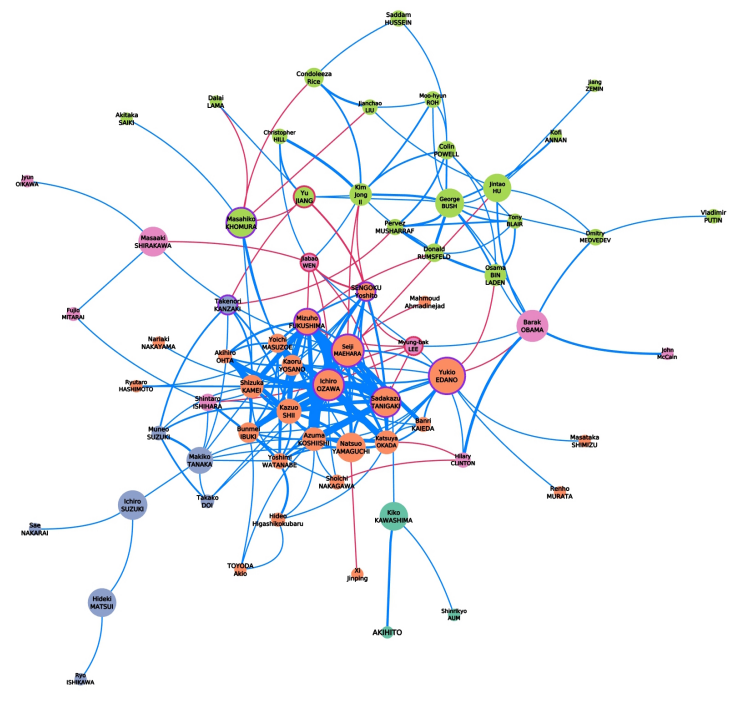

Fig. 8. Details readable on zoom. The network derived after filtering nodes from Fig. 7. Edges width encode the number of common topics. Red edges represent connections between Japanese and foreign politicians (otherwise blue). Node scolor correspond to the different Louvain clusters, from which we notice the Japanese (center in orange) and the international politicians (top in green). National politicians with strong 'foreign' links are circled in purple, and foreign politicians with strong 'national' links in dark red.

payed an active role in international matters by highlighting them (circled in purple in the Fig. 8, mostly at the right frontier of the orange community). We do so by counting the ratio by counting the number of their ties with international representatives and threshold them based on their cumulative probability distribution [31]. As a result, we find $Y$. Edano, $S$. Tanigaki, S. Maehara, M. Fukushima, Y. Sengoku, I. Ozawa, T. Kanzaki, M. Khomura. With the same process on the other side, we can identify (circled in red) Yu Jiang, Jiabao Wen, and Lee Myung-bak as having redundant apparition on topics with national politicians. The case of Lee Myung-Bak seems to have particularly raised a great interest among national politicians, totalizing 5 connections.

\section{Time slicing the topic network}

Thanks to well defined periods of time corresponding to PM cabinets, we can use topic segmentation as a support to observe not the overall network but each slice involving the persons' interactions over the different cabinets (Fig. 9).

To compare the political landscapes of each cabinet, we pick out the top 2 or 3 Japanese politicians in ranking of centrality and number of topics, who are neither a PM nor have been detected during the preceding cabinets. We then scan through all cabinets to verify in which cabinet the person has been detected or not. As a measure of 'political interaction', we can count the number of topics of each politician in which they have been detected with others during the cabinet. In total we have collected 21 prominent politicians, which will be used to compare cabinets one to another.

Based on this subset of $21+7$ PM we can finally estimate a rough (Jaccard) proximity between cabinets as shown in Fig. 9(1). The periods from Abe 1 to Noda known for the series of resigning PM, shows the highest proximity one to another, and interestingly to Koizumi 1. However, Koizumi's two following cabinets appear very different, suggesting that he set a very different media/politics scene during this time.

\section{SOME OBSERVATIONS}

This exploration led us to some understanding of the media/politics scene presented by NHK News 7. Based on this data, together with the knowledge we provide, the different PM stand out like no one else. They can be directly identified in all aspects of the data: first, purely quantitatively speaking, they occupy most of the media scene during their own cabinets; then, in the different networks, they also occupy a very central place; the different time-related analysis makes it especially obvious during their cabinets. We also learn by looking at individual PM: most of them show some level of activity before their mandate and we can observe two opposite cases. On one side, Abe is actually more central than Koizumi himself during Koizumi 3 (Fig. 9(d)). On the other side, Noda came 'out of nowhere' before becoming PM (Fig. 5). Despite of Hatoyama and Aso appearing quite strong nodes in the different networks, they never appeared on screen together (Fig. 6) even if they were heading two consecutive cabinets in period of time where the media/politics scene of consecutive cabinets is very similar - maybe because they are the leaders of two opposite parties.

A person by person analysis would be too long to detail in this paper, but the network exploration allows us to draw hypothesis on the important figures of the Japanese media scene at the different periods of time, then enabling a quick inspection of the individual video segments that qualify nodes and edges, to get the precise story.

Strikingly, one very particular politician comes out all along this study, I. Ozawa, who is (in)famously known as the "Shadow Shogun". Getting into details into Ozawa's role in the Japanese politics is a fascinating work on its own [32], but put in short, he is known for all the connections and roles he has played behind the scene, building alliances and often changing side - although never he became PM. To delve into this kind of details, the domain knowledge should be more precise, e.g. encoding the politicians' affiliations at time $t$. Nevertheless, we cannot get pass the fact that Ozawa is utterly present in the media. He is connected to different politicians through so many topics, making him a central figure over the 12 years observed.

Another very interesting point which is worth noting concerns the Imperial family. The Japanese Constitution forbids the Imperial family to take any part in politics, and observing the links surrounding the members of the family are of high interest to survey their actions. Our system finds very little connections (purple nodes in Fig. 9(c), (d), and (h)): they mostly concern the revision of the Imperial Household Law because of the issue concerning the succession to the Imperial Throne.

\section{DISCUSSION AND FUTURE WORK}

The different network views provide a powerful tool to understand the media situation, but we also need to draw the limits of the definition of these networks. As for now, the topic association brings the most meaningful construction of links, even if no actual semantics has yet been introduced in the system. It is equally important to understand how the 


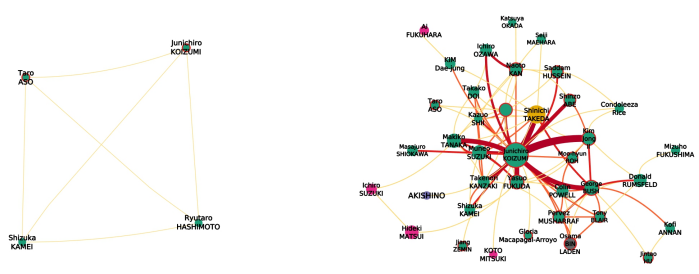

(a) Mori 2

2000/7/4 - 2001/4/26

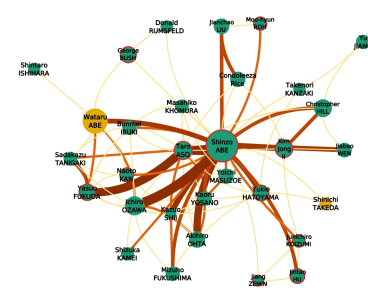

(e) Abe 1

2006/09/26 - 2007/09/26

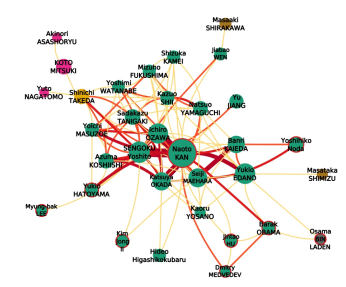

(i) Kan

2010/06/08 - 2011/09/02

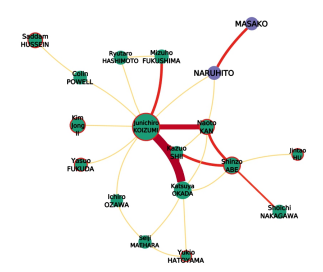

(c) Koizumi 2

2003/11/19 - 2005/09/21

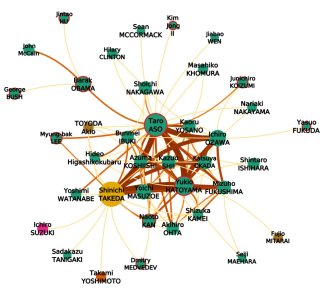

(g) Aso

2008/09/24 - 2009/09/16

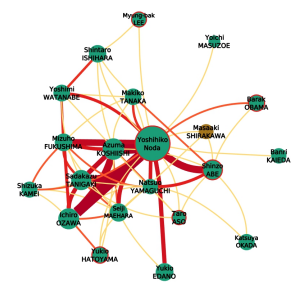

(j) Noda

2011/09/02 - 2012/12/26

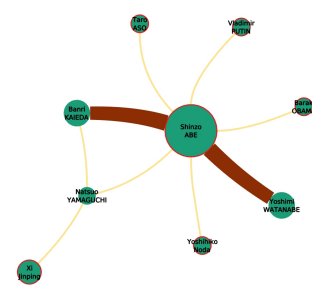

(k) Abe 2

2012/12/26 - 2014/12/24

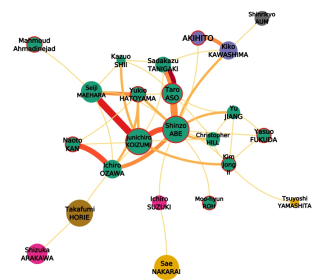

(d) Koizumi 3

2005/09/21 - 2006/09/26

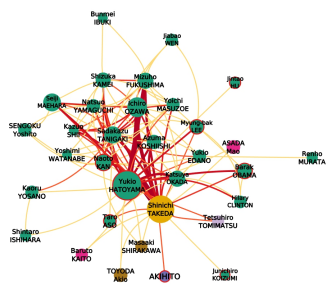

(h) Hatoyama

2009/09/16 - 2010/06/08

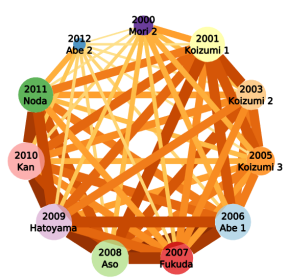

(1) Comparison of all cabinets

Fig. 9. The networks are better seen zoomed. From Mori 2 (a) to Abe 2 (k), the topic networks during the different cabinets with the same encoding as in Fig. 6. (1) This network shows the (Jaccard) proximity between cabinets given their most visible politicians on the NHK's scene. The size of nodes encodes the number of persons detected during a cabinet. The edges color and size encode the Jaccard proximity (the darker, the closer). We can notice how things have slowly changed from one cabinet to another during the 2006-2011 game of musical PM chairs.
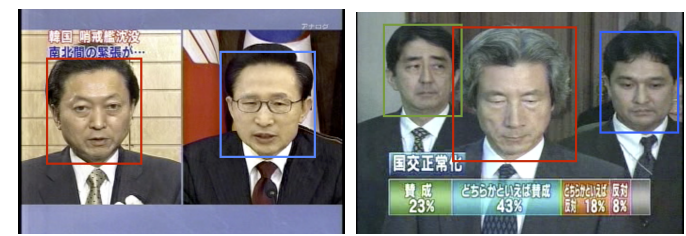

Fig. 10. The difference between screen co-occurence on a 'split screen' (Left) or within a same picture (Right) - Image courtesy of the NHK.

different pre-processing parts may have strong influences in later interpretations.

The screen overlap network has the strongest family of links in terms of social ties, but it is also the most subject to controversy in two ways. First, because of the bursty characteristics of our data, the limited but reliable subset of people, and parameters of our face-detections make the amount of screen co-detections limited. Then, because many detections concern split screens, which in in turn often means an opposition of ideas on a same subject, hence defining a sort of negative link - something we would like to investigate in the future. We want to distinguish this case from the screen co-occurence, which holds the different meaning of people standing in the same room at the same time (Fig. 10).

The same comment may also be made on the shot cooccurrence network, which finally extends the latter with a lighter meaning. For example, some shots occur behind the anchorman switching from one topic to another, sometimes leading to false positive links. Besides the system showing a good accuracy [25], some face occurrences may remain untracked, but we can still draw our conclusions thanks to the large period of time we observe.

Since we are discussing the data itself, our future work will extend the set of people to all faces detected in the dataset, not limited to the tagged individuals. We also put effort in enhancing the precision of the detection; and the addition of semantic information derived from the topic detection will be a great improvement.

This paper only scratches the surface, but the analysis of news data craves for application of many network analysis techniques. For example, the different overlaying families of links (screen, shot, topics) also form a multiplex network as in [33]. We can draw multiplex networks as in [15] with people interacting through cabinets and hopefully find cohesive groups of politicians. The dynamic of links is also of great interest and $\Delta$-cliques [34] (cliques over time in a stream of links) is a promising lead. In addition to finding outliers, we will be interested in groups of political actors who regularly appear together among similar topics. 


\section{CONCLUSION}

This work has introduced the production and analysis of face detection and tracking data over twelve years of news broadcast. We have detailed the data's characteristics and brought a few outliers. Together with the use of topic segmentation and some limited domain knowledge, we have derived many networks, each presenting a different point of view on the data. The combined views of these networks shows interesting insights on the story behind the data, an arguably clear picture of the media/politics landscape during the different cabinets, also isolating key players at different levels.

Rather different to the classical topic detection and tracking approaches of news data, this work brings up if not confirms the relevance of network analysis derived from news data. By itself, this is also an interesting framework for many potential contributions to the current challenges of social network analysis - including, but not limited to, multiplex and multi-attributed network analysis, dynamic networks, and their combination.

Finally, this work has given us useful directions that will help us design visualization tools, which we wish to put as quickly as possible in the hands of domain experts, sociologists and journalists, for an in-depth analysis of over 12 years of news.

\section{REFERENCES}

[1] A. J. Hey, S. Tansley, K. M. Tolle et al., The fourth paradigm: dataintensive scientific discovery. Microsoft Research Redmond, WA, 2009.

[2] J. Allan, Topic detection and tracking: event-based information organization. $\quad$ Springer Science \& Business Media, 2002, vol. 12.

[3] N. Hervé, M.-L. Viaud, J. Thièvre, A. Saulnier, J. Champ, P. Letessier, O. Buisson, and A. Joly, "Otmedia: the french transmedia news observatory," in Proceedings of the 21st ACM international conference on Multimedia. ACM, 2013, pp. 441-442.

[4] J. Cagé, "Media competition, information provision and political participation," Unpublished manuscript, Harvard University, 2014.

[5] P. Resnick, R. K. Garrett, T. Kriplean, S. A. Munson, and N. J. Stroud, "Bursting your (filter) bubble: Strategies for promoting diverse exposure," in Proceedings of the ACM 2013 Conference on Computer Supported Cooperative Work Companion, 2013, pp. 95-100.

[6] R. Hazell and B. Worthy, "Assessing the performance of freedom of information," Government Information Quarterly, vol. 27, no. 4, pp. 352-359, 2010.

[7] F. Schroff, D. Kalenichenko, and J. Philbin, "Facenet: A unified embedding for face recognition and clustering," arXiv preprint arXiv:1503.03832, 2015.

[8] P. Viola and M. J. Jones, "Robust real-time face detection," International journal of computer vision, vol. 57, no. 2, pp. 137-154, 2004.

[9] I. Ide and F. Nack, "Explain this to me!" ITE Transactions on Media Technology and Applications, vol. 1, no. 2, pp. 101-117, 2013.

[10] C. Castillo, G. De Francisci Morales, M. Mendoza, and N. Khan, "Says who?: automatic text-based content analysis of television news," in Proceedings of the 2013 international workshop on Mining unstructured big data using natural language processing. ACM, 2013, pp. 53-60.

[11] S. Sudhahar, R. Franzosi, and N. Cristianini, "Automating quantitative narrative analysis of news data." in WAPA, 2011, pp. 63-71.

[12] D. Lazer, "Networks in political science: Back to the future," PS: Political Science \& Politics, vol. 44, no. 01, pp. 61-68, 2011.

[13] L. A. Adamic and N. Glance, "The political blogosphere and the 2004 us election: divided they blog," in Proceedings of the 3rd international workshop on Link discovery. ACM, 2005, pp. 36-43.
[14] M. K. Martin, J. Pfeffer, and K. M. Carley, "Network text analysis of conceptual overlap in interviews, newspaper articles and keywords," Social Network Analysis and Mining, vol. 3, no. 4, pp. 1165-1177, 2013.

[15] B. Renoust, G. Melançon, and M.-L. Viaud, "Entanglement in multiplex networks: understanding group cohesion in homophily networks," in Social Network Analysis-Community Detection and Evolution. Springer, 2014, pp. 89-117.

[16] M. C. Waumans, T. Nicodème, and H. Bersini, "Topology analysis of social networks extracted from literature," PloS one, vol. 10, no. 6, p. e0126470, 2015.

[17] C.-J. Nan, K.-M. Kim, and B.-T. Zhang, "Social network analysis of tv drama characters via deep concept hierarchies," in Proceedings of ASONAM 2015, 2015.

[18] B. Mish, "Game of Nodes: A Social Network Analysis of Game of Thrones," 2015. [Online]. Available: https://gameofnodes.wordpress.com/

[19] M. Itoh, M. Toyoda, C. Z. Zhu, S. Satoh, and M. Kitsuregawa, "Image flows visualization for inter-media comparison," in Pacific Visualization Symposium (PacificVis), 2014 IEEE. IEEE, 2014, pp. 129-136.

[20] H. Luo, J. Fan, J. Yang, W. Ribarsky, and S. Satoh, "Analyzing largescale news video databases to support knowledge visualization and intuitive retrieval," in Visual Analytics Science and Technology, 2007. VAST 2007. IEEE Symposium on. IEEE, 2007, pp. 107-114.

[21] M.-L. Viaud, J. Thièvre, H. Goëau, A. Saulnier, and O. Buisson, "Interactive components for visual exploration of multimedia archives," in Proceedings of the 2008 international conference on Content-based image and video retrieval. ACM, 2008, pp. 609-616.

[22] C. Seifert, V. Sabol, W. Kienreich, E. Lex, and M. Granitzer, "Visual analysis and knowledge discovery for text," in Large-Scale Data Analytics. Springer, 2014, pp. 189-218.

[23] N. Katayama, H. Mo, I. Ide, and S. Satoh, "Mining large-scale broadcast video archives towards inter-video structuring," in Advances in Multimedia Information Processing-PCM 2004. Springer, 2005, pp. 489-496.

[24] I. Ide, H. Mo, N. Katayama, and S. Satoh, "Topic threading for structuring a large-scale news video archive," in Image and Video Retrieval. Springer, 2004, pp. 123-131.

[25] T. D. Ngo, H. T. Vu, L. Duy-Dinh, and S. Satoh, "Face retrieval in largescale news video datasets," IEICE TRANSACTIONS on Information and Systems, vol. 96, no. 8, pp. 1811-1825, 2013.

[26] G. Bradski et al., "The opencv library," Doctor Dobbs Journal, vol. 25, no. 11 , pp. 120-126, 2000.

[27] J. Shi and C. Tomasi, "Good features to track" in Computer Vision and Pattern Recognition, 1994. Proceedings CVPR'94., 1994 IEEE Computer Society Conference on. IEEE, 1994, pp. 593-600.

[28] X. Wang, C. Zhai, X. Hu, and R. Sproat, "Mining correlated bursty topic patterns from coordinated text streams," in Proceedings of the 13th ACM SIGKDD international conference on Knowledge discovery and data mining. ACM, 2007, pp. 784-793.

[29] V. D. Blondel, J.-L. Guillaume, R. Lambiotte, and E. Lefebvre, "Fast unfolding of communities in large networks," Journal of Statistical Mechanics: Theory and Experiment, vol. 2008, no. 10, p. P10008, 2008.

[30] S. B. Seidman, "Network structure and minimum degree," Social networks, vol. 5, no. 3, pp. 269-287, 1983.

[31] I. Herman, M. S. Marshall, and G. Melançon, "Density functions for visual attributes and effective partitioning in graph visualization," in Information Visualization, 2000. InfoVis 2000. IEEE Symposium on. IEEE, 2000, pp. 49-56

[32] I. Meyer, "The History of Japan podcast, Episode 82: The Shadow Shogun, Redux," 2014. [Online]. Available: https://historyofjapan.wordpress.com/2014/12/20/episode-82-theshadow-shogun-redux/

[33] M. Kivelä, A. Arenas, M. Barthelemy, J. P. Gleeson, Y. Moreno, and M. A. Porter, "Multilayer networks," Journal of Complex Networks, vol. 2, no. 3, pp. 203-271, 2014.

[34] J. Viard, M. Latapy, and C. Magnien, "Computing maximal cliques in link streams," arXiv preprint arXiv:1502.00993, 2015. 